\title{
Birt-Hogg-Dubé syndrome: a literature review and case study of a Chinese woman presenting a novel FLCN mutation
}

\author{
Shengyu Hao, Fei Long, Fenglan Sun, Teng Liu, Daowei Li and Shujuan Jiang*
}

\begin{abstract}
Background: The Birt-Hogg-Dubé (BHD) syndrome is a very rare autosomal dominant form of genodermatosis caused by germline mutations in the folliculin (FLCN) gene, which is mapped to the p11.2 region in chromosome 17. BHD commonly presents cutaneous fibrofolliculomas, pulmonary cysts, renal cell carcinoma, and recurrent pneumothoraxes. The disease is easily ignored or misdiagnosed as pneumothorax, pulmonary lymphangiomyomatosis (LAM), or emphysema. Follow-up and guidelines for managing recurrent pneumothoraxes in these patients are lacking.

Case presentation: We reported the case of a 56-year-old Chinese woman who presented skin lesions, multiple lung bubblae, recurrent pneumothoraxes, thyroid nodules, and pulmonary inflammatory pseudotumors (PITs). The patient had a family history of pneumothoraxes and renal tumor. The BHD diagnosis was confirmed by genetic testing, which revealed a novel FLCN mutation in exon 14. Furthermore, the patient underwent a bullectomy because of recurrent pneumothorax 6 years ago.

Conclusion: To our knowledge, the novel mutation in exon 14 and the manifestation of PIT in the present case have never been reported for BHD. The patient underwent a bullectomy previously with no relapse at the last follow-up before the preparation of this report, thereby suggesting that thoracotomy with bullectomy may be a possible therapeutic approach for some BHD patients with recurrent pneumothorax.
\end{abstract}

Keywords: Birt-Hogg-Dubé syndrome, Mutation, Lung bubblae, Pneumothorax, Treatment

\section{Background}

BHD syndrome is a rare autosomal dominant form of genodermatosis; the disease characterized by cutaneous fibrofolliculomas, pulmonary cysts, renal cell carcinoma, and recurrent pneumothoraxes [1]. Several other manifestations of BHD have been reported, such as colorectal carcinoma and colon polyps [2-4], thyroid nodules, parotid oncocytomas, multiple lipomas, oral papules, and cancer [5, 6]. However, their causal associations with BHD have not been clinically validated.

BHD was first reported as an inherited dermatological syndrome in 1977; the gene responsible for the syndrome was cloned in 2002 [7, 8]. To date, 152 unique pathogenic FLCN gene mutations in 616 families have been reported in worldwide; approximately $90 \%$ of these

\footnotetext{
* Correspondence: docjiangshujuan@163.com

Department of Respiratory Medicine, Shandong Provincial Hospital affiliated to Shandong University, 324 Jingwu Road, Jinan, Shandong 250021, China
}

mutations were reported in Europe and the United States [6]. The most frequent hot spot of mutation occurs in a tract of eight cytosines in exon 11 identified in approximately $50 \%$ of BHD patients regardless of ethnicity. Mutations in exons 9 and 12 are common, and their association with lung cysts and pneuomothoraces is statistically significant $[1,5,9]$. Multiple bilateral pulmonary cysts occur in 70 to $84 \%$ of the affected members in BHD families [5, 10, 11]. Approximately $30 \%$ of BHD patients will develop spontaneous pneumothoraxes, most frequently before the age of 40 years (median age of onset, 38 years) in Europe and the United States [10]. By contrast, multiple pulmonary cysts with recurrent pneumothorax are observed in approximately $90 \%$ of the BHD cases in Japanese patients [12]. Although the number of case reports describing lung disorders has increased, data are still lacking on the effective measures and follow-up to support the treatment of pneumothorax in BHD. 
We reported the case of a Chinese woman with skin lesions, multiple lung bullae, recurrent pneumothorax, thyroid nodes, PIT, and family history of spontaneous pneumothoraxes and renal tumor. The woman was subsequently diagnosed with a novel mutation. Moreover, the patient underwent a pulmonary bullectomy for recurrent pneumothorax 6 years ago.

\section{Case presentation}

A 56-year-old non-smoking Chinese woman visited our hospital; she has cough, expectoration, and throat discomfort for the past 45 days. The patient had no fever, chest congestion, or chest pain. Upon physical examination, multiple white dome-shaped papules were found on her cheek, palpebra, fossa axillaris, and back (Fig. 1). Her medical history showed a tendency to illness. The patient first developed pneumothorax at the age of 36, with multiple bilateral pulmonary cysts and subsequently underwent a tube thoracostomy. After 12 years, the pneumothorax recurred. At 50 years of age, the patient had a third pneumothorax; her right lung was compressed by $40 \%$. Finally, the patient consented to surgical intervention for therapeutic purposes. During surgery, thin-walled bullae with sizes from $0.1 \mathrm{~cm}$ to $3.0 \mathrm{~cm}$ in diameter were observed on the right lung pleura. The bulla over $10 \mathrm{~cm}$ in diameter was found in the right horizontal fissure, and a $2.5 \mathrm{~cm}$-diameter intrapulmonary bulla containing liquid was found in the lateral segment of the right middle lobe. Partial pleura adhesions were released by an electrosurgical generator. The $10 \mathrm{~cm}$ bulla was completely removed through wedge-shaped excision. The other bubblae on the

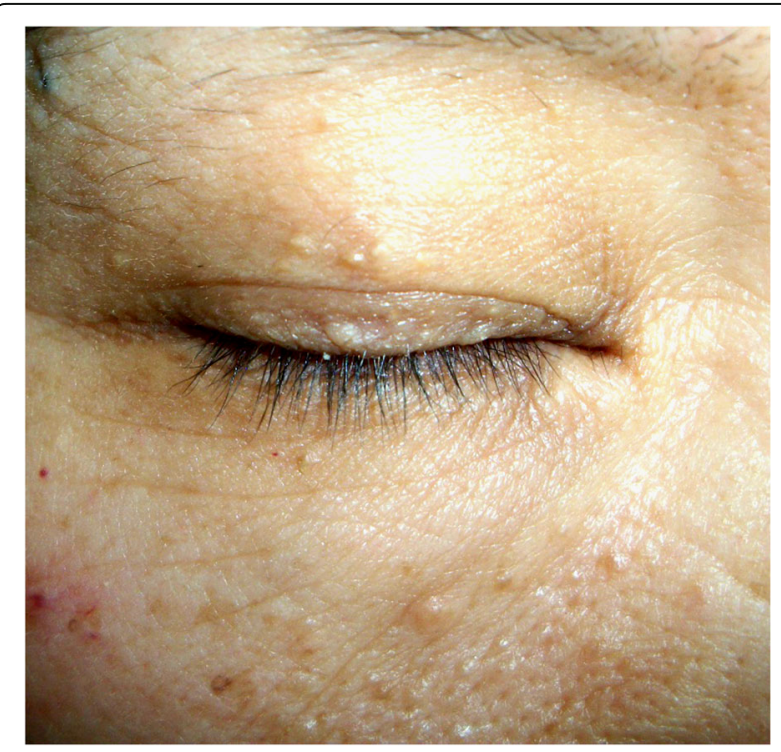

Fig. 1 Multiple pale and dome-shaped macules over the patient's face, which are characteristic skin lesions of BHD syndrome pleura were clamped, closed and removal. The incisions were sutured, and the thoracic wall was rubbed and congested for pleurodesis. Histopathological examination showed that the inner surfaces of the cysts were lined with epithelial cells positive for CK7 and TTF-1; the cysts occasionally contained internal septa consisting of alveolar walls (Fig. 2).

The patient is a non-smoker, who easily became ill even when she was young. Furthermore, she had a family history of spontaneous pneumothorax. The mother and maternal aunt of the patient had histories of pulmonary bullae and pneumothoraxes; her mother died of renal cancer at the age of 71 , with pulmonary bubblae and recurrent pneumothorax. Her 31-year-old daughter and 69-year-old male cousin were classified as BHDunaffected because they did not show manifestations of BHD upon physical examinations. Her maternal aunt died in her 80s without tumor diseases, but with several recurrent pneumothoraxes. A chest computed tomography $(\mathrm{CT})$ scan taken at our hospital showed multiple cystic lesions throughout the lungs, mainly in the basilar regions (Fig. 3). A $2.8 \mathrm{~cm} \times 2.3 \mathrm{~cm}$ solid lesion was present in the posterior basal segement of the lower right lobe (Fig. 4), which was subsequently proven to be a PIT by ultrasound guided percutaneous lung biopsy.

Biopsy revealed massive infiltrations of inflammatory cells, including plasma cells and lymphocytes admixed with fibroblasts and myofibroblasts (Fig. 5). Immunohistochemical studies showed positive reactions for CD3, CD20, and Ki-67, whereas no staining was observed with antibodies against CEA, IgG4, IgG, and ALK. These findings were reported as fibrous changes at the same location in the CT performed previously (Fig. 4a, c). Ultrasound examination showed that the patient did not develop a renal carcinoma, but multiple thyroid nodules had emerged.

Given that the patient had multiple skin papules, cystic lesions, pneumothorax, and a family history of pneumothorax and renal tumor, we suspected a congenital disease, particularly BHD. Therefore, we performed blood DNA analysis, and four mutations were identified (Table 1). A mutation of $\mathrm{T}$ to $\mathrm{C}(\mathrm{c} .2297 \mathrm{~T}>\mathrm{C})$ in exon 14 leads to the change from Phe to Ser (p.Phe766Ser). To our knowledge, this mutation has never been reported before (Fig. 6). Besides the mutation in exon 14, we also detected a mutation in exon 1 (c.-299C > T) with a mutation rate of 0.42 . Another mutation in intron $8(\mathrm{c} .871+36 \mathrm{G}>\mathrm{A})$, with a mutation rate of 0.17 , causes the codon 303 mutation (p.G ly303Arg) in FLCN 2 transcription. The third mutation in intron 9 (c.1062 $+6 \mathrm{C}>\mathrm{T})$ has mutation rate of 0.66 . However, these three mutations were previously reported to have a minimal correlation to the onset of BHD [13]. 


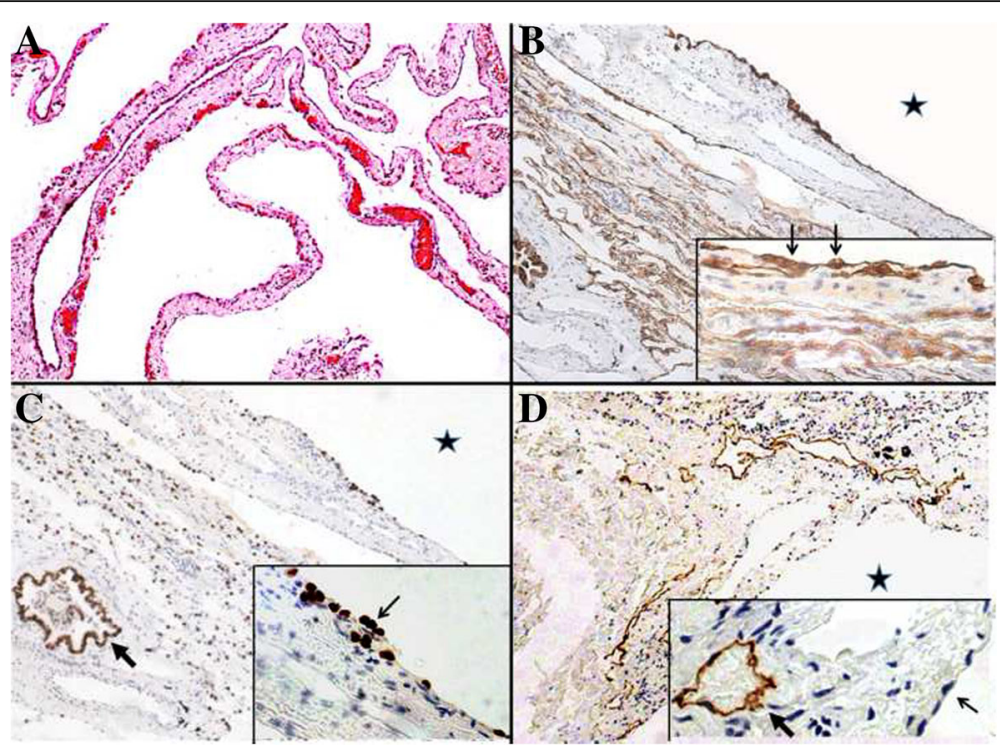

Fig. 2 Histopathological features of the resected lung specimens from the patient 6 years ago. Cysts are indicated by stars. a These cysts are incorporated with interstitial stroma of the interlobular septum or pleura (hematoxylin-eosin-safranin staining). Cysts lined by $\mathbf{b} C K 7^{+}$and c $T \mathrm{~F}-1^{+}$pneumocytes. $\mathbf{d}$ D2-40 is negative in the lining (thin arrows) but positive in lymphatics (thick arrow)

\section{Discussion and conclusion}

BHD syndrome is difficult to diagnose at initial evaluation because of its low morbidity and variable manifestations. BHD is not yet well-known in East Asia, particularly in China. BHD is a rare type of genodermatosis and an autosomal dominant disease caused by FLCN mutations. To date, $90 \%$ of the reported BHD patients are from Europe and the United States; 82 to 92\% of the cases in these regions have characteristic skin symptoms. By contrast, the rate of characteristic symptoms is 20 to $29 \%$ in Japanese patients, which may account for the relative difficulty to recognize the disease; the clinical manifestations of BHD may have potential racial differences $[6,14,15]$. Recurrent pneumothorax is observed in approximately $90 \%$ of Asian patients [12], which is higher by $60 \%$ than in Western countries.

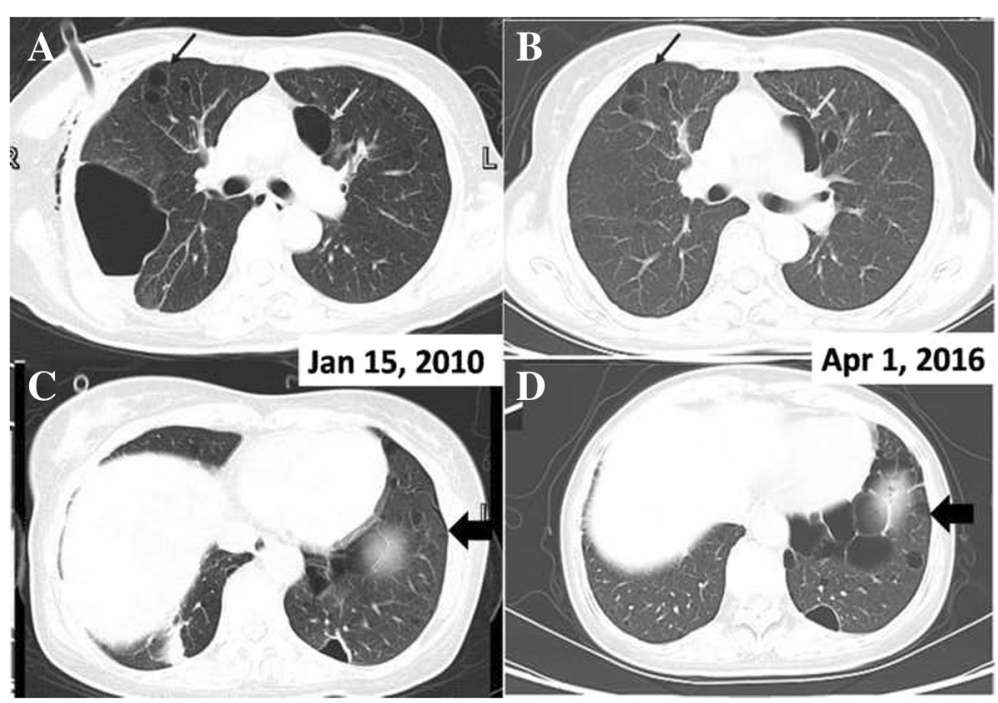

Fig. 3 Postoperative changes are shown in the right lung. a, c The previous CT scan shows numerous cysts in the lung bases; one dominant cyst measures $8.4 \mathrm{~cm} \times 5.1 \mathrm{~cm}$ in the right lung and contains some liquid; another huge cyst is anterior to the heart (white arrow). $\mathbf{b}$, d The right lung expanded well after the operation with no obvious scar or atelectasis. Some cysts remained stable (black arrow) or became even smaller (white arrow). However, more cysts appeared in the lung bases (thick arrow) 


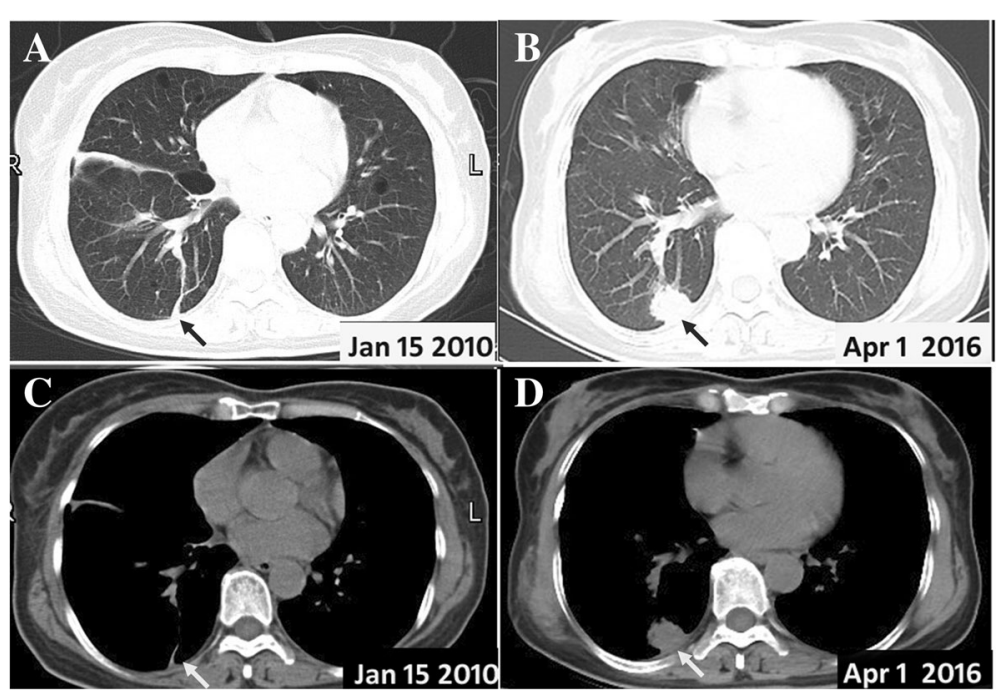

Fig. 4 Chest CT scan with pulmonary pseudotumor. a Previous CT scan shows a fibrous change in the same slice. $\mathbf{b}$ The present CT scan shows a $2.8 \mathrm{~cm} \times 2.3 \mathrm{~cm}$ pulmonary solid lesion with irregular margins and adjacent pleural thickness. $\mathbf{c}$, $\mathbf{d}$ Corresponding mediastinal window

However, BHD is easily misdiagnosed as cystic lung disease, particularly LAM, Langerhans cell histiocytosis, and lymphocytic interstitial pneumonia.

Imaging studies may facilitate differential diagnosis. Cysts in BHD cases are round or oval, withthin, visible, and uniform walls. The cysts are typically located in the basilar and mediastinal lung regions, which differ from the typical apical location of smoking-related emphysema and bullae in primary spontaneous pneumothoraxes [16]. The number and size of the cysts are variable; cysts less than $1 \mathrm{~cm}$ in diameter are most commonly seen [17]. We confirmed these findings in the present patient (Fig. 3). LAM is the most difficult to differentiate from BHD, particularly when associated with cutaneous and renal involvement. Cysts in BHD and LAM are thin walled, but those in LAM are smaller, more circular, and equally distributed $[16,18]$. On the other hand, the prognosis of BHD is better. LAM usually progresses to respiratory insufficiency with increasing lung cysts. However, BHD typically does not result in respiratory failure.

Little is known about the development of pulmonary cysts in BHD patients. Some reviews suggest that numerous cysts appear to remain stable or unruptured because the frequency of pneumothorax in elderly BHD cases is not as high as that in middle-aged patients [19]. In the present case, we compared the CT findings. Some bullae of the right lung were removed by bullectomy 5 years ago without any scars or atelectasis in the recent $\mathrm{CT}$. The changes were evident in the lungs. Some bullae remained stable, whereas others were absorbed as they shrank or disappeared. However, bullae at the bottom became larger (Fig. 3). The pathological findings of the cysts in BHD are non-specific [20], which is also true in the present case (Fig. 2). Almost 15 years have passed since the discovery of FLCN, and increasing numbers of BHD cases have been reported. However, follow-up of these affected patients is still needed.

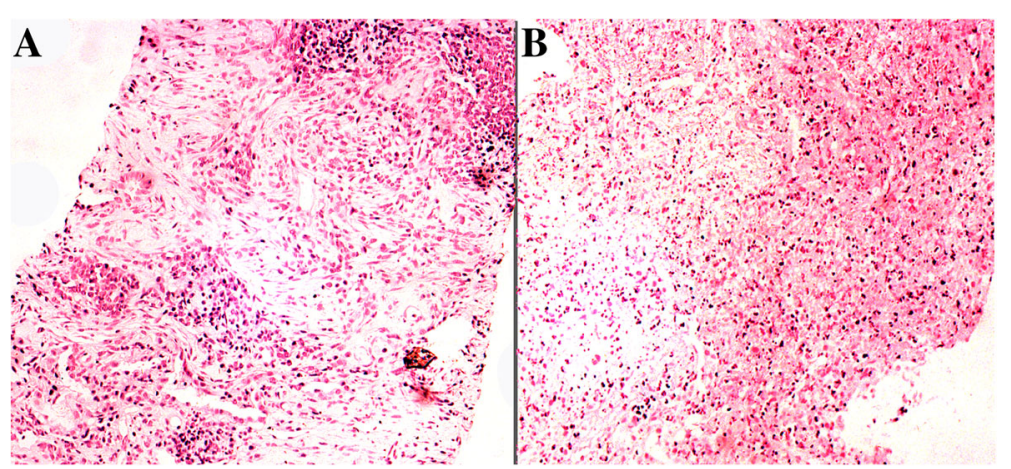

Fig. 5 Ultrosound-guided needle biopsy of the lung lesion with immunohistochemistry showed that it is positive for an inflammatory pesudotumor with $\mathbf{a}$ extensively hyperplasic fibrous and inflammatory cell infiltration, and $\mathbf{b}$ necrosis lung tissues 
Table 1 Summary of total mutations identified in the FLCN gene by sequencing

\begin{tabular}{|c|c|c|c|c|c|}
\hline dbSNP & mRNA position ${ }^{a}$ & Location or $\triangle A A^{a}$ & Alleles $^{a}$ & Minor Allele & MAF in $\mathrm{CHB}$ \\
\hline rs1708629 & c.-299 & $5^{\prime} U T R$ & $C>T$ & C & 0.42 \\
\hline rs3744124 & $c .871+36$ & Intron 8 & $G>A$ & G & 0.17 \\
\hline rs8065832 & c. $1062+6$ & Intron 9 & $C>T$ & C & 0.66 \\
\hline p.F766S & c.2297 & Exon 14 & $T>C$ & T & None \\
\hline
\end{tabular}

$\triangle A A$ change in amino acid, MAF minor allele frequency, $C H B$ samples from Han Chinese in Beijing, China studied in Hapmap project

a Referenced to the positive strand and folliculin isoform 1 [GenBank: NM_144997]

Our case report described a novel mutation in FLCN, and a new manifestation of PIT in lung lesions of BHD. Previous studies showed that $F L C N$ serves as a tumor suppressor gene. The germline mutations in FLCN are related to $\mathrm{BHD}$ pathogenesis. However, clear genotypephenotype associations have not been reported in BHD $[4,21]$. PIT is an uncommon benign lesion in the lungs; this lesion is considered to be an inflammatory or reactive lesion rather than a neoplasm. The exact etiology is unknown, and an immune disorder seems to be an acceptable hypothesis. In the present patient, the poor condition of the lungs, with multiple cysts, recurrent pneumothoraxes, and tube thoracostomy, disturbed the pulmonary immunity, thereby making it easily infected. However, these conditions also exist in other BHD patients who did not develop a PIT. The only difference is that the patient in the present case has a novel mutation in FLCN. Given that the exact etiology of PIT is unknown and no exact genotypephenotype associations of BHD have been reported, more data are still needed.

The correlation of the new manifestation in this patient with the new mutation has not been confirmed.
The patient also showed multiple thyroid nodules, which were also reported in almost half of the BHD patients. This patient did not develop any renal tumors at the last follow-up. The patient's mother died of a renal tumor. Renal tumors are present in $27 \%$ of the BHD patients [21]. Therefore, we informed the patient of the possibility that she could be affected by a renal tumor and suggested that she undergo abdominal ultrasound scan or CT annually.

The occurrence of multiple bilateral pulmonary cysts and spontaneous pneumothorax is high in BHD patients $[5,10,11]$. The management of BHD from a pulmonary perspective is focused on the treatment of pneumothorax. Conservative measures (observation, aspiration, and tube thoracostomy) are taken to manage the initial pneumothorax, which is usually undiagnosed during the first instance. However, BHD patients still face a high risk of recurrent pneumothorax, thereby making these patients anxious. Video-assisted thoracoscopy and mechanical or chemical pleurodesis are typically reserved for recurrent or non-healing cases [16, 22], which make it difficult for reoperation and thoracic surgeries in later years. A unique method was reported to

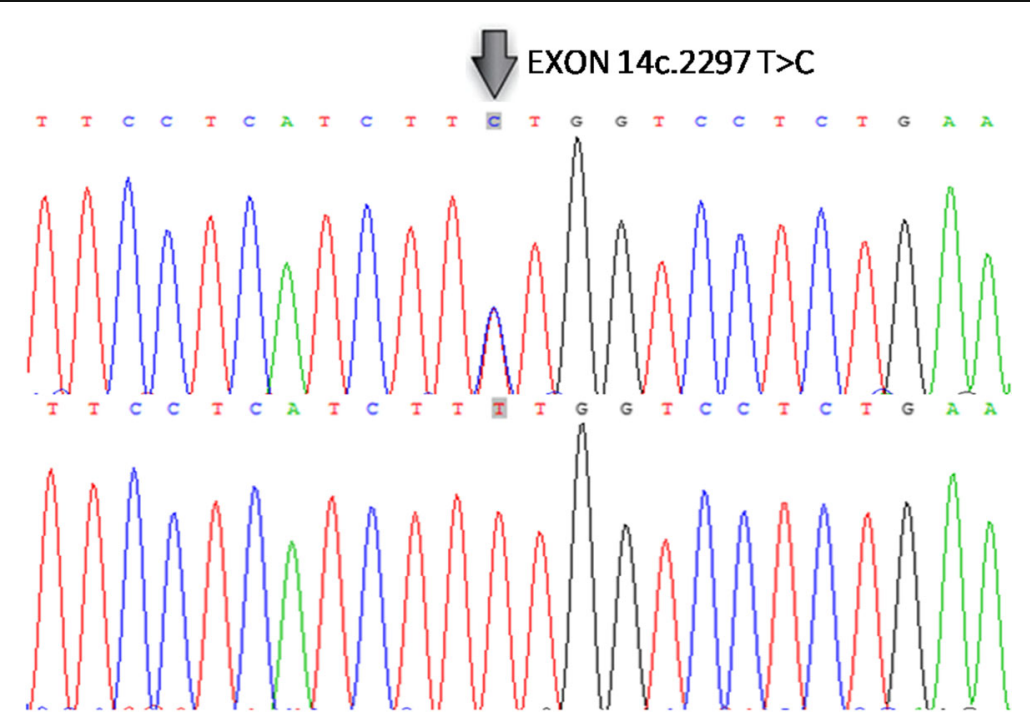

Fig. 6 Identification of a germline heterozygous FLCN mutation in the patients. The result showed a heterozygous mutation in exon 14 resulting in an animo acid transformation of phenylalanine into serine (p.Phe766Ser) 
prevent recurrent pneumothorax without adhesion to the chest wall, as well as total or partial pleural covering; however, success is heavily dependent on local expertise $[23,24]$. Although the number of case reports on lung disorders has increased, data collection on effective measures and follow-up are still lacking to support the treatment of pneumothorax in BHD.

We reported the case of a Chinese woman who underwent pulmonary bulla resection 6 years ago and did not have a recurrent pneumothorax at the last follow-up. Adequate management of bullae on the pulmonary surface, thoracic wall congestion, and hemorrhage during surgery favored the adhesion between the visceral and parietal pleura, thereby contributing to the success of the operation.

\section{Abbreviations}

BHD: Birt-Hogg-Dubé; CT: A chest computed tomography; FLCN: Folliculin; LAM: Lymphangiomyomatosis; PIT: Pulmonary inflammatory pseudotumor

\section{Acknowledgements}

We would like to thank Dr. Zhou Wang (Department of Pathology, Shandong Provincial Hospital) for performing the mutation analysis of the BHD gene and valuable assistance in the pathological diagnosis.

\section{Funding}

This work was supported in part by National Natural Science Foundation (No. 81370138) and Shandong provincial Key Research and Development Program (No. 2015GSF118028).

\section{Availability of data and materials}

Not applicable.

\section{Authors' contributions}

SH analyzed and interpreted the patient data, drafted the manuscript and made the figures. FL, SJ and FS performed the histological examination, proposed the study and revised the manuscript. TL, DL and SJ have made substantial contributions to analysis and interpretation of patient data. All authors read and approved the final manuscript.

\section{Competing interests}

The authors declare that they have no competing interests.

\section{Consent for publication}

Written informed consent was obtained from patients for the publication of this case report and any accompanying images.

\section{Ethics approval and consent to participate}

Written informed consent was obtained from patients for the publication of this case report and any accompanying images.

\section{Received: 10 July 2016 Accepted: 28 January 2017}

Published online: 21 February 2017

\section{References}

1. Menko FH, van Steensel MA, Giraud S, Friis-Hansen L, Richard S, Ungari S, Nordenskjold M, Hansen TV, Solly J, Maher ER. Birt-Hogg-Dube syndrome: diagnosis and management. Lancet Oncol. 2009;10(12):1199-206.

2. Hornstein OP, Knickenberg M. Perifollicular fibromatosis cutis with polyps of the colon-a cutaneo-intestinal syndrome sui generis. Arch Dermatol Res. 1975;253(2):161-75.

3. Rongioletti F, Hazini R, Gianotti G, Rebora A. Fibrofolliculomas, tricodiscomas and acrochordons (Birt-Hogg-Dube) associated with intestinal polyposis. Clin Exp Dermatol. 1989;14(1):72-4.

4. Nahorski MS, Lim DH, Martin L, Gille JJ, McKay K, Rehal PK, Ploeger HM, van Steensel $M$, Tomlinson IP, Latif $F$, et al. Investigation of the Birt-Hogg-Dube tumour suppressor gene (FLCN) in familial and sporadic colorectal cancer I Med Genet. 2010;47(6):385-90.

5. Toro JR, Wei MH, Glenn GM, Weinreich M, Toure O, Vocke C, Turner M, Choyke P, Merino MJ, Pinto PA, et al. BHD mutations, clinical and molecular genetic investigations of Birt-Hogg-Dube syndrome: a new series of 50 families and a review of published reports. J Med Genet. 2008;45(6):321-31.

6. The BHD Foundation. https://www.bhdsyndrome.org/, Accessed 2011.

7. Birt AR, Hogg GR, Dube WJ. Hereditary multiple fibrofolliculomas with trichodiscomas and acrochordons. Arch Dermatol. 1977;113(12):1674-7.

8. Nickerson ML, Warren MB, Toro JR, Matrosova V, Glenn G, Turner ML, Duray P, Merino $M$, Choyke P, Pavlovich CP, et al. Mutations in a novel gene lead to kidney tumors, lung wall defects, and benign tumors of the hair follicle in patients with the Birt-Hogg-Dube syndrome. Cancer Cell. 2002;2(2):157-64.

9. Toro JR, Pautler SE, Stewart L, Glenn GM, Weinreich M, Toure O, Wei MH, Schmidt LS, Davis L, Zbar B, et al. Lung cysts, spontaneous pneumothorax, and genetic associations in 89 families with Birt-Hogg-Dube syndrome. Am J Respir Crit Care Med. 2007;175(10):1044-53.

10. Schmidt LS, Nickerson ML, Warren MB, Glenn GM, Toro JR, Merino MJ, Turner ML, Choyke PL, Sharma N, Peterson J, et al. Germline BHD-mutation spectrum and phenotype analysis of a large cohort of families with BirtHogg-Dube syndrome. Am J Hum Genet. 2005;76(6):1023-33.

11. Kluger N, Giraud S, Coupier I, Avril MF, Dereure O, Guillot B, Richard S, Bessis D. Birt-Hogg-Dube syndrome: clinical and genetic studies of 10 French families. Br J Dermatol. 2010;162(3):527-37.

12. Kunogi M, Kurihara M, Ikegami TS, Kobayashi T, Shindo N, Kumasaka T, Gunji Y, Kikkawa M, Iwakami S, Hino O, et al. Clinical and genetic spectrum of BirtHogg-Dube syndrome patients in whom pneumothorax and/or multiple lung cysts are the presenting feature. J Med Genet. 2010;47(4):281-7.

13. Cho MH, Klanderman BJ, Litonjua AA, Sparrow D, Silverman EK, Raby BA. Folliculin mutations are not associated with severe COPD. BMC Med Genet. 2008:9:120.

14. Murakami Y, Wataya-Kaneda M, Tanaka M, Takahashi A, Tsujimura A, Inoue K, Nonomura N, Katayama I. Two Japanese cases of birt-hogg-dube syndrome with pulmonary cysts, fibrofolliculomas, and renal cell carcinomas. Case Rep Dermatol. 2014;6(1):20-8.

15. Vernooij M, Claessens T, Luijten M, van Steensel MA, Coull BJ. Birt-HoggDube syndrome and the skin. Fam Cancer. 2013:12(3):381-5.

16. Gupta N, Seyama K, McCormack FX. Pulmonary manifestations of Birt-HoggDube syndrome. Fam Cancer. 2013;12(3):387-96.

17. Dal Sasso AA, Belem LC, Zanetti G, Souza CA, Escuissato DL, Irion KL, Guimaraes MD, Marchiori E. Birt-Hogg-Dube syndrome. State-of-the-art review with emphasis on pulmonary involvement. Respir Med. 2015;109(3):289-96.

18. Tomassetti S, Carloni A, Chilosi M, Maffe A, Ungari S, Sverzellati N, Gurioli C, Casoni G, Romagnoli M, Gurioli C, et al. Pulmonary features of Birt-HoggDube syndrome: cystic lesions and pulmonary histiocytoma. Respir Med. 2011;105(5):768-74

19. Furuya M, Nakatani Y. Birt-Hogg-Dube syndrome: clinicopathological features of the lung. J Clin Pathol. 2013:66(3):178-86.

20. Fabre A, Borie R, Debray MP, Crestani B, Danel C. Distinguishing the histological and radiological features of cystic lung disease in Birt-HoggDube syndrome from those of tobacco-related spontaneous pneumothorax. Histopathology. 2014;64(5):741-9.

21. Zbar B, Alvord WG, Glenn G, Turner M, Pavlovich CP, Schmidt L, Walther M, Choyke P, Weirich G, Hewitt SM, et al. Risk of renal and colonic neoplasms and spontaneous pneumothorax in the Birt-Hogg-Dube syndrome. Cancer Epidemiol Biomarkers Prev. 2002;11(4):393-400.

22. Haynes D, Baumann MH. Management of pneumothorax. Semin Respir Crit Care Med. 2010;31(6):769-80.

23. Kurihara M, Kataoka H, Ishikawa A, Endo R. Latest treatments for spontaneous pneumothorax. Gen Thorac Cardiovasc Surg. 2010;58(3):113-9.

24. Okada A, Hirono T, Watanabe T, Hasegawa G, Tanaka R, Furuya M. Partial pleural covering for intractable pneumothorax in patients with Birt-HoggDube Syndrome. Clin Respir J. 2015. [Epub ahead of print]. 